\title{
Acute COVID-19 Infection in a Pediatric Patient with ROHHAD
}

\author{
Daniel S. Udrea ${ }^{10}$ Merrick Lopez ${ }^{2}$ Michael Avesar ${ }^{2}$ Sonea Qureshi ${ }^{2}$ Anthony Moretti ${ }^{2}$ \\ Shamel A. Abd-Allah ${ }^{2}$ Harsha K. Chandnani ${ }^{2}$
}

1 Department of Emergency Medicine, Loma Linda University Medical Center, Loma Linda, California, United States

2 Division of Pediatric Critical Care, Department of Pediatrics, Loma Linda University Children's Hospital, Loma Linda, California, United States

\begin{abstract}
Address for correspondence Harsha K. Chandnani, MD, MBA, MPH, Department of Pediatric Critical Care, Loma Linda University Children's Hospital, 11175 Campus Street, Suite A1117, Loma Linda, CA 92354, United States (e-mail: hchandnani@llu.edu).
\end{abstract}

J Pediatr Genet 2022;11:309-312.

\begin{abstract}
Keywords

- ROHHAD

- COVID-19

- inflammatory syndrome

The novel coronavirus (severe acute respiratory syndrome coronavirus-2) has led to a global pandemic. In the adult population, coronavirus disease 2019 (COVID-19) has been found to cause multiorgan system damage with predicted long-term sequelae. We present a case of a 10-year-old boy with a history of ROHHAD (rapid-onset obesity with hypothalamic dysregulation, hypoventilation, and autonomic dysregulation) who presented with hypoxia, emesis, and abdominal pain. Imaging found bilateral ground glass opacities in the lungs and a pericardial effusion. Laboratory evaluation was concerning for elevated inflammatory markers. Remdesivir, hydroxychloroquine, and anticoagulation (heparin and enoxaparin) were utilized. The patient's severe respiratory failure was managed with conventional mechanical ventilation, inhaled nitric oxide, and airway pressure release ventilation. We hope that this report provides insight into the course and management of the severe acute pediatric COVID-19 patient, specifically with underlying comorbidities such as ROHHAD. Clinical trial registration is none.
\end{abstract}

\section{Introduction}

The rates of hospitalization and mortality of severe acute respiratory syndrome coronavirus-2 (SARS-CoV-2) infection across the globe are increasing. There is a modicum of literature that describes the management of coronavirus disease 2019 (COVID-19) in pediatric patients. Features such as elevated serum ferritin and bilateral abnormalities on chest computed tomography (CT) are commonly described in adult literature but poorly described in pediatric literature. ${ }^{1}$ We describe a case of a 10 -year-old boy with ROHHAD (rapid-onset obesity with hypothalamic dysregulation, hypoventilation, and autonomic dysregulation) presenting with COVID-19 and highlight his initial presentation, clinical course, challenges with his comorbidities, and management strategies leading to a successful hospital discharge.

\section{Case}

A 10-year-old boy with ROHHAD (rare disorder of abnormalities associated with the autonomic nervous system, endocrine system, and respiratory control), morbid obesity (weight: $95 \mathrm{~kg}$, body mass index: 45.2), severe obstructive sleep apnea requiring nocturnal continuous positive airway pressure, and intellectual delay, presented with 1 day of abdominal pain and nonbilious, blood-tinged vomiting. ${ }^{2}$ On presentation to the emergency department, he was found to be afebrile, tachycardic (heart rate 113 beats per minute), hypoxic (oxygen saturation 50\%), and in respiratory distress. Initially, oxygen saturations improved with nasal cannula. CT of the chest demonstrated a pericardial effusion measuring $1.8 \mathrm{~cm}$ in thickness, bilateral pulmonary ground glass infiltrates, pulmonary congestion, and hepatosplenomegaly (-Fig. 1). Initial received

July 17,2020

accepted after revision

September 14, 2020

published online

October 26, 2020 (c) 2020. Thieme. All rights reserved.

Georg Thieme Verlag KG,

Rüdigerstraße 14,

70469 Stuttgart, Germany
DOI https://doi.org/ 10.1055/s-0040-1718874. ISSN 2146-4596. 


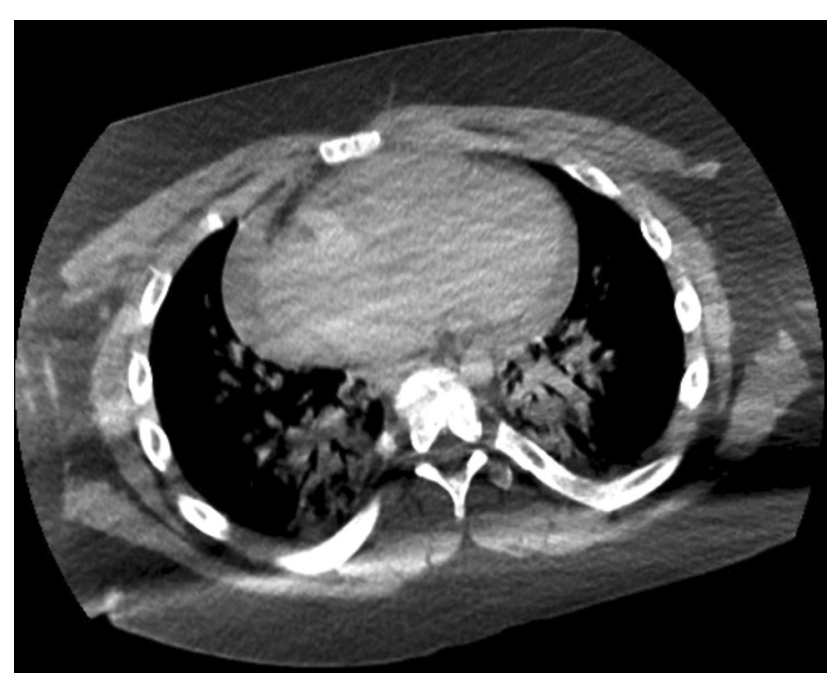

Fig. 1 Computed tomography of the chest demonstrating ground glass opacities, pericardial effusion.

laboratories revealed leukopenia and an elevated $\mathrm{N}$-terminal probrain natriuretic peptide of $1,038 \mathrm{pg} / \mathrm{L}$. He was suspected to have acute congestive heart failure and was transferred to our children's hospital for further management.

The patient was initially admitted to the pediatric stepdown intensive care unit in a negative pressure room, pending COVID-19 testing. Approximately 4 hours after arrival, the patient was escalated to high-flow nasal cannula (HFNC) and transferred to the pediatric intensive care unit for worsen respiratory distress. He was febrile up to $104.2^{\circ} \mathrm{F}$. Chest X-ray (CXR) was concerning for alveolar and interstitial pulmonary edema at the lung bases (-Fig. 2). Intravenous furosemide was administered for radiographic and clinical evidence of pulmonary edema. Dexmedetomidine drip was started for agitation and vasopressor support for hypotension. His respiratory viral panel resulted negative, while his COVID-19 testing (DiaSorin nasopharyngeal swab polymerase chain reaction test) resulted positive.

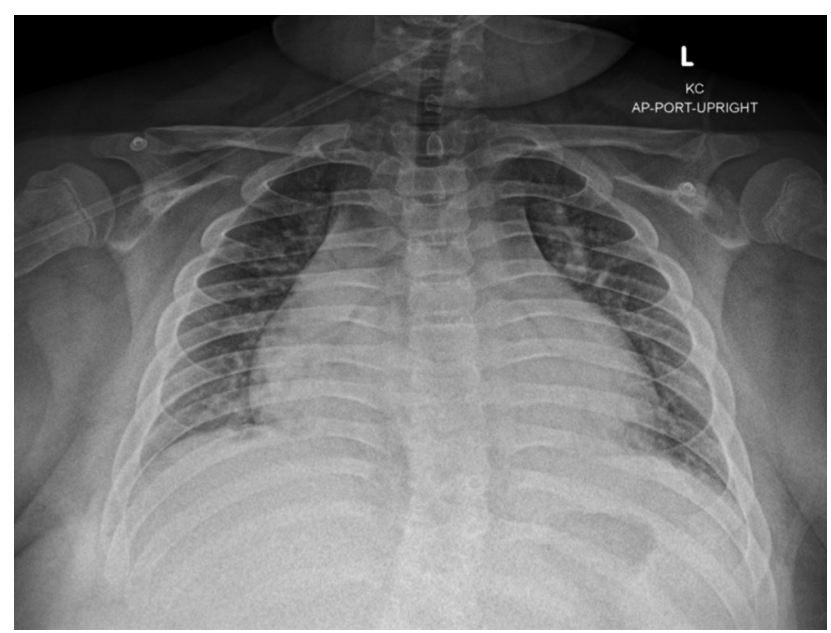

Fig. 2 Chest X-ray demonstrating alveolar and interstitial pulmonary edemas.
He was intubated due to progressive hypoxic respiratory failure with rapid sequence intubation using ketamine, succinylcholine, and direct laryngoscopy. Full personal protective equipment (including an intubation hood) was used with minimal essential personnel present. Endotracheal, blood, and urine cultures were sent, and broad-spectrum antibiotics were started for persistent fever and leukopenia. Infectious disease recommended starting hydroxychloroquine as well as remdesivir, which was requested from Gilead pharmaceuticals for compassionate use. Heparin drip was initiated for a presumed hypercoagulable state associated with COVID-19. A milrinone infusion was also started for afterload reduction in the setting of acute

Table 1 Initial serum laboratory values on admission to the pediatric ICU

\begin{tabular}{|l|l|}
\hline WBC & $12.9 \mathrm{bil} / \mathrm{L}$ \\
\hline HGB & $9.2 \mathrm{~g} / \mathrm{dL}$ \\
\hline HCT & $29.6 \%$ \\
\hline Platelets & $145 \mathrm{bil} / \mathrm{L}$ \\
\hline PT & $17.9 \mathrm{~s}$ \\
\hline INR & $1.5 \mathrm{n} / \mathrm{a}$ \\
\hline PTT & $49.8 \mathrm{~s}$ \\
\hline ESR & $36 \mathrm{~mm} / \mathrm{h}$ \\
\hline Na & $141 \mathrm{mMol} / \mathrm{L}$ \\
\hline K & $3.7 \mathrm{mMol} / \mathrm{L}$ \\
\hline Cl & $102 \mathrm{mMol} / \mathrm{L}$ \\
\hline CO 2 & $27 \mathrm{mMol} / \mathrm{L}$ \\
\hline Anion gap & $12 \mathrm{n} / \mathrm{a}$ \\
\hline BUN & $17 \mathrm{mg} / \mathrm{dL}$ \\
\hline Creatinine & $1.1 \mathrm{mg} / \mathrm{dL}$ \\
\hline Albumin & $4.2 \mathrm{~g} / \mathrm{dL}$ \\
\hline AST & $151 \mathrm{U} / \mathrm{L}$ \\
\hline ALT & $56 \mathrm{U} / \mathrm{L}$ \\
\hline Alk phos & $55 \mathrm{U} / \mathrm{L}$ \\
\hline T. bilirubin & $0.6 \mathrm{mg} / \mathrm{dL}$ \\
\hline LD & $429 \mathrm{U} / \mathrm{L}$ \\
\hline CRP & $11.5 \mathrm{mg} / \mathrm{dL}$ \\
\hline Ferritin & $295 \mathrm{ng} / \mathrm{mL}$ \\
\hline Lactate & $3.8 \mathrm{mMol} / \mathrm{L}$ \\
\hline Pro-BNP & $3,347 \mathrm{pg} / \mathrm{mL}$ \\
\hline Troponin-T & $0.02 \mathrm{ng} / \mathrm{mL}$ \\
\hline IL-6 & $91.9 \mathrm{pg} / \mathrm{mL}$ \\
\hline Procalcitonin & $10.13 \mathrm{\mu g} / \mathrm{L}$ \\
\hline
\end{tabular}

Abbreviations: ALT, alanine aminotransferase; Alk phos, alkaline phosphatase; AST, aspartate aminotransferase; BUN, blood urea nitrogen; CRP, C-reactive protein; ESR, erythrocyte sedimentation rate; $\mathrm{HCT}$, hematocrit; $\mathrm{HGB}$, hemoglobin; ICU, intensive care unit; IL, interleukin; INR, international normalized ratio; LD, lactate dehydrogenase; PT, prothrombin time; PTT, partial thromboplastin time; WBC, white blood cell. 
congestive heart failure. Initial laboratories were concerning for transaminitis and elevated inflammatory markers. His initial laboratories on admission are listed in - Table $\mathbf{1}$. Echocardiogram showed a small globally distributed pericardial effusion with normal biventricular function, no evidence of elevated pulmonary artery pressure, and an ejection fraction of $63 \%$.

On day 2 of admission, the patient developed bloodtinged oral, nasal, and respiratory secretions; therefore, the heparin was held. Patient was continued on hydroxychloroquine. On day 3 , he was no longer febrile. Subcutaneous enoxaparin was started due to concern for hypercoagulability with persistently elevated D-dimers. Remdesivir was initiated (dose: $200 \mathrm{mg}$ first day, followed by $100 \mathrm{mg}$ daily for 9 additional days) and hydroxychloroquine was discontinued. On day 4, the patient began to have transient episodes of hypoxia and hypercarbia with no change in CXR. Inhaled nitric oxide (iNO) was initiated for persistent hypoxemia and suspected elevated pulmonary vascular resistance. On day 5 of admission, the patient's sodium level rose to $162 \mathrm{mMol} / \mathrm{L}$. Urine was dilute (specific gravity 1.002) and serum osmolality was elevated (344 $\mathrm{mOsm} / \mathrm{kg}$ ). Free water flushes were initiated. Due to declining $\mathrm{PaO}_{2} / \mathrm{FiO}_{2}(\mathrm{P} / \mathrm{F}$ ) ratio (221 to 138 ), the patient was prone, resulting in rapid significant improvement in $\mathrm{P} / \mathrm{F}$ ratio to 428.

On day 6, the patient's sodium remained elevated at 167 $\mathrm{mMol} / \mathrm{L}$ despite free water flushes. The patient's hypotension improved and epinephrine was titrated off. He was weaned off iNO with improving oxygenation and enteral feeds were initiated. Patient returned to supine position after intermittent proning. The next 2 days saw further increases in sodium levels (peak at $170 \mathrm{mMol} / \mathrm{L}$ ) and D-amino D-arginine vasopressin (DDAVP) was started for suspected diabetes insipidus, resulting in normalization of sodium levels over the next 7 days.

On day 9 , the patient's hemodynamics were very difficult to control. His $\mathrm{P} / \mathrm{F}$ ratios worsened, and proning and iNO were reinitiated, showing improvement in $\mathrm{P} / \mathrm{F}$ (240 to 316).

On day 15 of admission, the patient's fevers returned $\left(102.1^{\circ} \mathrm{F}\right)$ secondary to methicillin-sensitive Staphylococcus aureus pneumonia, requiring antibiotics. Over the next 3 days, the patient tolerated ventilator weaning trials and was successfully extubated to HFNC by day 19 of admission.
He was subsequently weaned to room air, transitioned to enteral medications, and discharged from the hospital after a 23-day hospital stay (-Fig. 3).

\section{Discussion}

To our knowledge, this is the first described case of COVID-19 infection in a patient with ROHHAD. These children are seemingly normal before developing hyperphagia and subsequent rapid-onset weight gain early in childhood. Over time, these patients may develop endocrine abnormalities resulting in sodium and water imbalances, and elevated prolactin and cortisol levels. The rapid weight gain is believed to contribute to the onset of obstructive sleep apnea and alveolar hypoventilation. Autonomic findings may manifest, including altered pupillary responses, changes in intestinal motility, temperature dysregulation, and bradycardia. At this time, no specific cause for ROHHAD has been found.,

At the time of this patient's hospital discharge, the World Health Organization reported more than 3.5 million global cases of COVID-19 with more than 200,000 deaths, including 75 pediatric deaths. ${ }^{5}$ Even as the number of cases grows, there is only a modicum of infectious, epidemiologic, and clinical data available to assist in the treatment of COVID-19positive pediatric patients. To date, the most common presentation of the acute pediatric COVID-19 patient ranges from completely asymptomatic to symptoms of an acute upper respiratory tract infection to gastrointestinal symptoms with progression to respiratory failure, coagulopathy, acute renal injury, and shock in severe cases. ${ }^{6}$

There were several challenges in this patient's management throughout his hospitalization, such as abnormal ventilation and oxygenation, labile hemodynamics, and hypernatremia. Upon intubation, several modes of ventilation were attempted, but we settled on airway pressure release ventilation as an effective mode for alveolar recruitment, especially with his heavy body habitus, short status, and propensity for hypoventilation. ${ }^{7}$ Proning and iNO also proved to be effective adjuncts for improving oxygenation. ${ }^{8}$ Prone positioning has been used in the management of hospitalized COVID-19 patients, has decreased mortality in adults with severe lung disease, and is currently being studied in pediatric trials. ${ }^{9,10}$

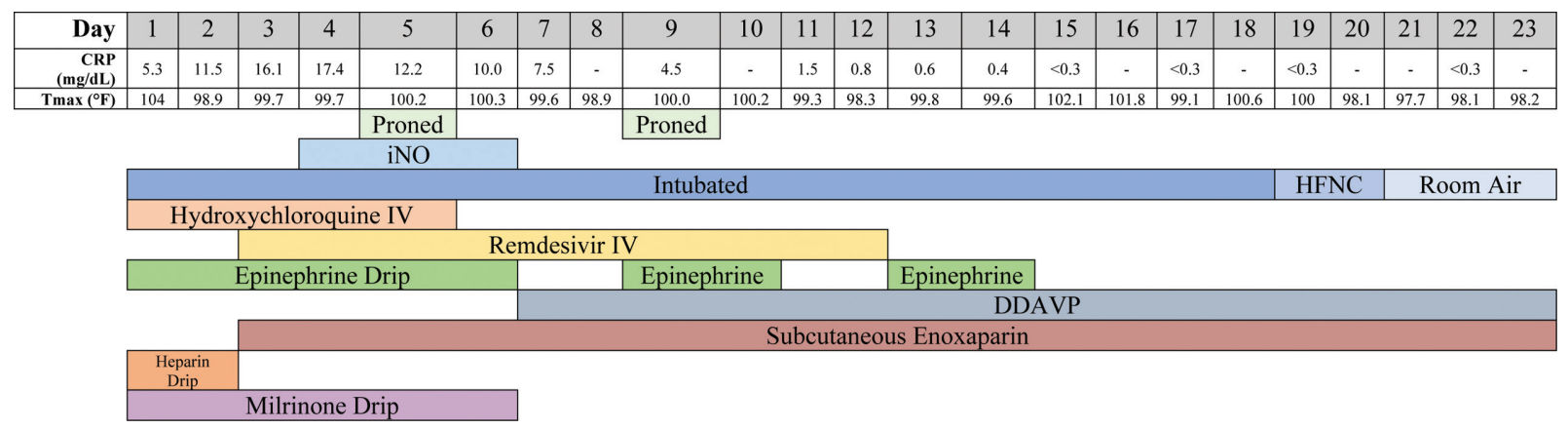

Fig. 3 Trends and treatments throughout hospital course. All dates that have no lab value correspond to the sample not being drawn that day at the clinician's direction. CRP, C-reactive protein; DDAVP, D-amino D-arginine vasopressin; HFNC, high-flow nasal cannula; iNO, inhaled nitric oxide; IV, intravenous. 
The patient's hemodynamics were also difficult to control, likely due to a combination of reasons, such as his underlying propensity for autonomic dysregulation. This was evidenced later in the patient's ICU course by the presence of bradycardia while off dexmedetomidine, and persistent constipation.

The patient's sodium was difficult to control despite adequate free water administration, possibly secondary to hypothalamic dysfunction as part of the patient's underlying genetic abnormality. The hypothalamus is responsible for the formation of arginine vasopressin in the posterior pituitary which functions to regulate extracellular fluid volume. The administration of DDAVP provided a significant improvement in the patient's hypernatremia and suggests a diagnosis of diabetes insipidus.

Laboratory tests, including but not limited to the D-dimer and serum ferritin, were trended throughout the patient's care as clinically indicated to monitor for the laboratory findings of COVID-19 sequelae that has been seen in the adult population. D-dimer, fibrinogen, and prothrombin time/ partial thromboplastin time testing were utilized to monitor the development of thrombosis despite anticoagulation therapy. Serum ferritin, C-reactive protein, procalcitonin, and lactate dehydrogenase testing were utilized to evaluate the development of rheumatologic and systemic inflammatory manifestations such as cytokine storm, and to detect possible superimposed bacterial infection.

Hospital protocol upon admission required the utilization of the COVID-19 DiaSorin (RNA) test with an approximate turnaround time of 2 hours. During the patient's ICU encounter, both nasopharyngeal and sputum samples were obtained and sent weekly to Mayo Clinic (RNA). Only the nasopharyngeal swabs returned with a positive result. Serologies returned positive for both immunoglobulin (Ig)G and IgM antibodies.

After the patient's initial COVID-19 RNA test returned positive, hydroxychloroquine and zinc supplementation were initiated based on preliminary reports available at the time suggesting viral load reduction in COVID-19 patients. ${ }^{11}$ However, recent literature suggests no benefit with hydroxychloroquine administration and it is unclear if our patient improved after its use. ${ }^{12}$ Remdesivir, an adenosine analog that inhibits viral replication with included antiviral activity against SARS-CoV-2, was administered under compassionate use, also with an unclear role in this patient's improvement. ${ }^{13}$ Preliminary evidence from a case series involving adult patients suggests clinical improvement but there has not been a randomized controlled trial to this date. ${ }^{14}$

\section{Conclusion}

We report a case of severe pediatric SARS-CoV-2 in the United States in a patient with a complex genetic condition. Our patient's course was complicated by respiratory failure, hemodynamic instability, endocrine abnormalities, and autonomic dysregulation. This case report may serve as a reference for other critical care clinicians tasked with caring for complex pediatric patients who are infected with COVID19. It is imperative for further literature to better describe the clinical presentation, course, and treatment of COVID-19 in the pediatric population.

\section{Authors' Contributions}

D.S.U. and H.K.C. conceptualized the report, drafted the initial manuscript, and assisted in revisions. M.L., M.A., S.Q., A.M., and S.A.A.-A. extracted and assembled the relevant data from the patient's hospital course, critically reviewed the manuscript for important intellectual content, and revised the manuscript. All authors contributed directly in the patient's care for an extended period of time. All authors approved the final manuscript as submitted and agree to be accountable for all aspects of the work.

\section{Conflict of Interest}

None declared.

\section{References}

1 Sinha IP, Harwood R, Semple MG, et al. COVID-19 infection in children. Lancet Respir Med 2020;8(05):446-447

2 Barclay SF, Rand CM, Nguyen L, et al. ROHHAD and Prader-Willi syndrome (PWS): clinical and genetic comparison. Orphanet J Rare Dis 2018;13(01):124

3 Ize-Ludlow D, Gray JA, Sperling MA, et al. Rapid-onset obesity with hypothalamic dysfunction, hypoventilation, and autonomic dysregulation presenting in childhood. Pediatrics 2007;120(01): e179-e188

4 Rapid-onset Obesity with Hypothalamic Dysfunction, Hypoventilation, and Autonomic Dysregulation. Accessed May 15, 2020 at: rarediseases.org

5 Centers for Disease Control and Prevention Provisional COVID-19 Death Counts by Sex, Age, and State. Accessed May 11, 2020 at: https://data.cdc.gov/NCHS/Provisional-COVID-19-Death-Countsby-Sex-Age-and-S/9bhg-hcku

6 Dong Y, Mo X, Hu Y, et al. Epidemiology of COVID-19 among children in China. Pediatrics 2020; 145(06):e20200702

7 Daoud EG. Airway pressure release ventilation. Ann Thorac Med 2007;2(04):176-179

8 Guérin C, Reignier J, Richard J-CPROSEVA Study Group, et al. Prone positioning in severe acute respiratory distress syndrome. $\mathrm{N}$ Engl J Med 2013;368(23):2159-2168

9 Moghadam VD, Shafiee H, Ghorbani M, Heidarifar R. Prone positioning in management of COVID-19 hospitalized patients. Braz J Anesthesiol 2020;70(02):188-190

10 U.S. National Library of Medicine PROSpect: Prone and Oscillation Pediatric Clinical Trial. Accessed May 11, 2020 at: https://clinicaltrials.gov/ct2/show/NCT03896763. Accessed May 11, 2020

11 Gautret P, Lagier J-C, Parola P, et al. Hydroxychloroquine and azithromycin as a treatment of COVID-19: results of an open-label non-randomized clinical trial. Int J Antimicrob Agents 2020;56 (01):105949

12 Chen J, Liu D, Liu L, et al. A pilot study of hydroxychloroquine in treatment of patients with moderate COVID-19 [in Chinese]. Zhejiang Da Xue Xue Bao Yi Xue Ban 2020;49(02):215-219

13 Wang M, Cao R, Zhang L, et al. Remdesivir and chloroquine effectively inhibit the recently emerged novel coronavirus (2019-nCoV) in vitro. Cell Res 2020;30(03):269-271

14 Grein J, Ohmagari N, Shin D, et al. Compassionate use of remdesivir for patients with severe Covid-19. N Engl J Med 2020;382 (24):2327-2336 